\title{
Fast and accurate method for flexible ligand superposition and shapebased screening
} M Sastry ${ }^{2}$, J Duan*1, W Sherman ${ }^{2}$ and S Dixon ${ }^{2}$

Address: 1Schrödinger GmbH, Dynamostrasse 13, 68165 Mannheim, Germany and 2Schrödinger Inc, 120 West 45th Street, 29th Floor, New York, NY 10036, USA

* Corresponding author

from 4th German Conference on Chemoinformatics

Goslar, Germany. 9-II November 2008

Published: 5 June 2009

Chemistry Central Journal 2009, 3(Suppl I):OI9 doi:I0.II86/I752-I53X-3-SI-OI9

This abstract is available from: http://www.journal.chemistrycentral.com/content/3/SI/OI9

(C) 2009 Sastry et al; licensee BioMed Central Ltd.

The use of 3-dimensional molecular shapes has been demonstrated to be useful in comparing small molecules. We have developed a novel method that allows for rapid superposition and scoring of flexible molecules.

The core algorithm is based on the alignment of a set of optimal atom triads followed by volume overlap scoring. The method can process approximately 1000 conformations per second on a modern computer. For flexible superposition we demonstrate on a large data set of crystal structures how accurate alignments can be obtained rapidly (less than one second per molecule) in an automated fashion. Next, we apply the method to virtual screening and show high enrichment rates across a broad range of targets and ligands. A one million compound database (100 conformations per compound) can be processed in approximately 15 minutes on a 100 processor cluster, making this method attractive for pre-screening large databases before downstream pharmacophore-based or docking screens. 